\title{
Potential of Arbidol for Post-exposure Prophylaxis of COVID-19 Transmission: A Preliminary Report of a Retrospective Cohort Study
}

\author{
Jin-nong ZHANG ${ }^{1 \dagger}$, Wen-jing WANG ${ }^{1 \dagger}$, Bo $\mathrm{PENG}^{2}$, Wei $\mathrm{PENG}^{3}$, Yi-sheng ZHANG ${ }^{4}$, Ya-ling WANG ${ }^{5}$, Yan WAN ${ }^{5}$, \\ Jiang $\mathrm{CHANG}^{6}$, Ling MAO ${ }^{5}$, Xiao-ping MIAO ${ }^{6}$, Ya-nan $\mathrm{LI}^{5 \#}$, Yi-fan ZHOU ${ }^{5 \#}$, Bo $\mathrm{HU}^{5 \#}$ \\ ${ }^{I}$ Department of Emergency Medicine, Union Hospital, Tongji Medical College, Huazhong University of Science and Technology, \\ Wuhan 430022, China \\ ${ }^{2}$ Peng Consulting Group, Salt Lake City 84108, UT, USA \\ ${ }^{3}$ Salt Lake Regional Medical Center, Heart and Lung Institute of Utah, Salt Lake City 84108, UT, USA \\ ${ }^{4}$ School of Materials Science and Engineering, Huazhong University of Science and Technology, Wuhan 430074, China \\ ${ }^{5}$ Department of Neurology, Union Hospital, Tongji Medical College, Huazhong University of Science and Technology, Wuhan \\ 430022, China \\ ${ }^{6}$ Department of Epidemiology and Biostatistics, Key Laboratory for Environment and Health, School of Public Health, Huazhong \\ University of Science and Technology, Wuhan 430030, China
}

(C) Huazhong University of Science and Technology 2020

\begin{abstract}
Summary: The efficient transmission of severe acute respiratory syndrome-2 coronavirus (SARSCoV-2) from patients to health care workers or family members has been a worrisome and prominent feature of the ongoing outbreak. On the basis of clinical practice and in-vitro studies, we postulated that post-exposure prophylaxis (PEP) using Arbidol is associated with decreased infection among individuals exposed to confirmed cases of COVID-19 infection. We conducted a retrospective cohort study on family members and health care workers who were exposed to patients confirmed to have SARS-CoV-2 infection by real-time RT-PCR and chest computed tomography (CT) from January 1 to January 16, 2020. The last follow-up date was Feb. 26, 2020. The emergence of fever and/or respiratory symptoms after exposure to the primary case was collected. The correlations between post-exposure prophylaxis and infection in household contacts and health care workers were respectively analyzed. A total of 66 members in 27 families and 124 health care workers had evidence of close exposure to patients with confirmed COVID-19. The Cox regression based on the data of the family members and health care workers with Arbidol or not showed that Arbidol PEP was a protective factor against the development of COVID-19 (HR 0.025, $95 \%$ CI $0.003-0.209, P=0.0006$ for family members and HR $0.056,95 \%$ CI $0.005-0.662, P=0.0221$ for health care workers). Our findings suggest Arbidol could reduce the infection risk of the novel coronavirus in hospital and family settings. This treatment should be promoted for PEP use and should be the subject of further investigation.
\end{abstract}

Key words: Arbidol; COVID-19; SARS-CoV-2; post-exposure prophylaxis

Literature and clinical evidence indicate that person-to-person transmission of severe acute respiratory syndrome coronavirus 2 (SARS-CoV-2), the pathogen that causes coronavirus disease 2019 (COVID-19), is highly efficient. A sample of 1099 patients with laboratory-confirmed COVID-19 in China delineated that $31.30 \%$ had been to Wuhan and $71.80 \%$ had contacted with people from Wuhan ${ }^{[1]}$. The

Jin-nong ZHANG, E-mail: zhangjnwhhb@163.com; Wenjing WANG, E-mail: wwj2010yx@163.com

†The authors contributed equally to this work.

\#Corresponding authors, Bo HU, E-mail: hubo@mail.hust. edu.cn; Yi-fan ZHOU, E-mail: yifan0180@hust.edu.cn; Yanan LI, E-mail: liyn@mail.hust.edu.cn transmission of COVID-19 in families is very efficient; in a report of a family with 6 members who travelled to Wuhan, 5 were identified as infected with COVID-19 ${ }^{[2]}$. The disease transmission aboard the well-known Diamond Princess cruise ship is potentially a model for understanding the efficiency of SARS-CoV-2 transmission in a dense hospital building inhabited by thousands of patients and health care workers. More than 3600 passengers were stranded on the cruise ship when the primary case was confirmed of COVID-19 on February 1, 2020. Following a 14-day quarantine period, a total of 624 people tested positive for SARSCoV-2 (https://www.google.com/amp/s/amp.cnn.com/ cnn/2020/02/18/asia/japan-health-guidelines- 
coronavirus-hnk-intl/index.html). Quarantine and postexposure prophylaxis (PEP) are options to prevent disease transmission. The well-known PEP for influenza infections is Oseltamivir ${ }^{[3]}$, however, there is no such pharmacologic agent demonstrated to prevent COVID-19 transmission after unprotected exposure to infected individuals. Arbidol (Umifenovir) is a broad-spectrum anti-viral agent and shown to be active against a large number of DNA/RNA and enveloped/ non-enveloped viruses ${ }^{[4]}$, which is widely used in China because of its use in treating influenza and recently its potential efficacy in treating COVID-19. Several in vitro studies indicate that Arbidol possesses inhibitory effect on coronavirus ${ }^{[5-7]}$ and its derivative, Arbidol mesylate is even stronger against SARS-CoV ${ }^{[7]}$. One study indicated that the therapeutic index of Arbidol (or selective index) on coronaviridae was 8.5 , which is much higher than for orthomyxoviridae, which are 2.4 and 2.5 for influenza A/Aichi/2/68(H3N2) and B/Beijing/184/93, respectively ${ }^{[5]}$. Clinical trials on the efficacy of Arbidol on COVID-19 are ongoing. Given the increasingly awareness of Arbidol, health care workers and members of the public are turning to Arbidol for both treatment and PEP. We conducted a retrospective cohort study to evaluate the potential of post-exposure prophylaxis with Arbidol in reducing transmission of SARS-CoV-2.

\section{MATERIALS AND METHODS}

\subsection{Definition and Identification of Cases and Controls}

We analyzed two cohorts: family members and health care workers. In the first cohort, each of 27 families had one primary case who was confirmed COVID-19. All of the 27 primary cases of family cohort were mild at the time of visit based on the newly released guideline from the Chinese government. Family members lived in the same apartment and had household contact with the primary cases without vigilance before the primary case developed symptoms. After confirmation of the primary case's infection, the family members sought medical attention. The families included in the analysis had consulted our hospital regarding PEP and potential treatment options of COVID-19 (table1).

The second cohort comprised of 124 health care workers in Wuhan Union Hospital initially exposed to a cluster of COVID-19 infected colleagues without standard respiratory protection. All of the source patients (the primary cases and the cluster of health care workers) were infected between January 1 and January 16, 2020.

\subsection{Data Collection}

For all participants, we collected personal demographic information, basic physical condition [specifically the presence of chronic comorbidities such as hypertension, diabetes, vascular disease, obesity defined by body mass index (BMI) $>30$ ], how and where the primary case was diagnosed, whether or not other family members or the health care workers were on PEP and if so, who in the families were on PEP, the medication used, dosage and duration of treatment. We also inquired for the emergence of fever and/or respiratory symptoms after exposure to the primary case. The diagnostic confirmation of COVID-19 was determined by reverse transcription quantitative realtime PCR (RT-PCR) on sputum sample or throat swab as described in recent literature ${ }^{[8,9]}$ and the coexistence of viral-like pneumonia on chest $\mathrm{CT}$.

Our study was approved by Medical Ethics Committee of Tongji Medical College of Huazhong University of Science and Technology, performed in accordance with the ethical standards established in the 1975 Declaration of Helsinki.

\subsection{Statistical Analysis}

We excluded all primary cases in the analysis. Chi-square test and Cox regression were applied using R 3.3.0. Continuous variables were expressed as mean \pm standard deviation (SD), and categorical variables as number. $P<0.05$ was defined as statistically significant. Missing information was assigned as a separate variable of "unknown" and the subjects were not removed from the Cox regression.

\section{RESULTS}

\subsection{General Characteristics of Cases and Controls}

We surveyed 27 families who had one primary case of COVID-19 infection and collected data from 66 family members, among them, 45 of 52 family members from 22 families took Arbidol and 14 family members from 5 families did not (table 1). Collectively, 45 family members used Arbidol PEP (table 2) and 1 became infected (table 3 ), while 21 family members did not use Arbidol and 12 became infected (table $3)$. The univariate analysis showed no significant differences in gender $(P=0.252)$, age $(P=0.628)$, chronic comorbidities $(P=0.659)$ or adult and children distribution $(P=0.057)$ between Arbidol group and non-Arbidol group (table 2).

We analyzed data from 124 health care workers (table 2), of whom 55 were given Arbidol PEP and 1 became infected, while 69 did not use Arbidol and 7 became infected (table 4). There was significant difference in gender $(P=0.002)$, profession (doctor or nurse) distribution $(P<0.001)$ and work place $(P<0.001)$ between Arbidol group and non-Arbidol group (table 2). Only 1 out of 13 confirmed COVID-19 family members took Arbidol (table 3), and only 1 out of 8 confirmed COVID-19 health workers took Arbidol (table 4). 


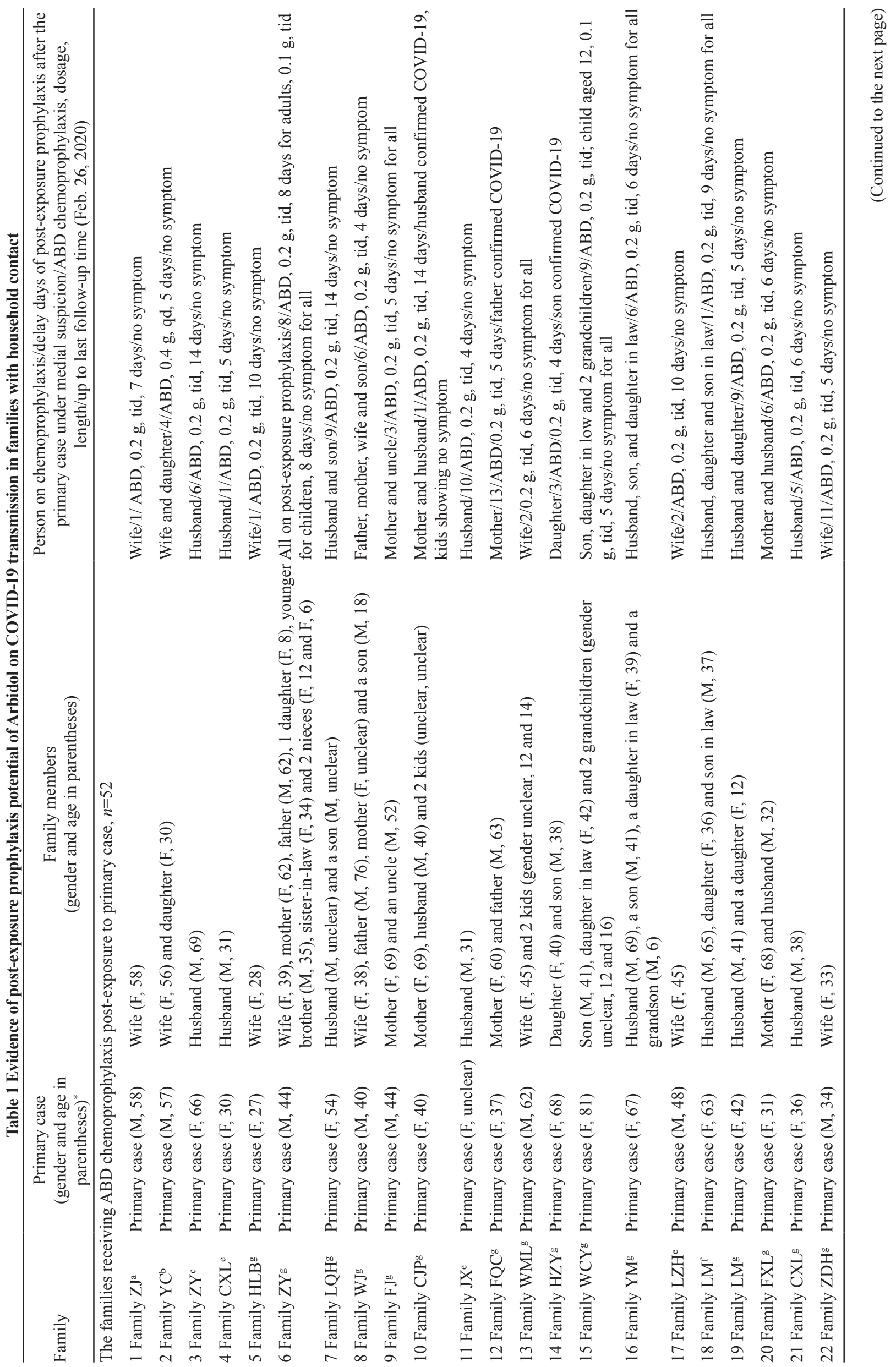




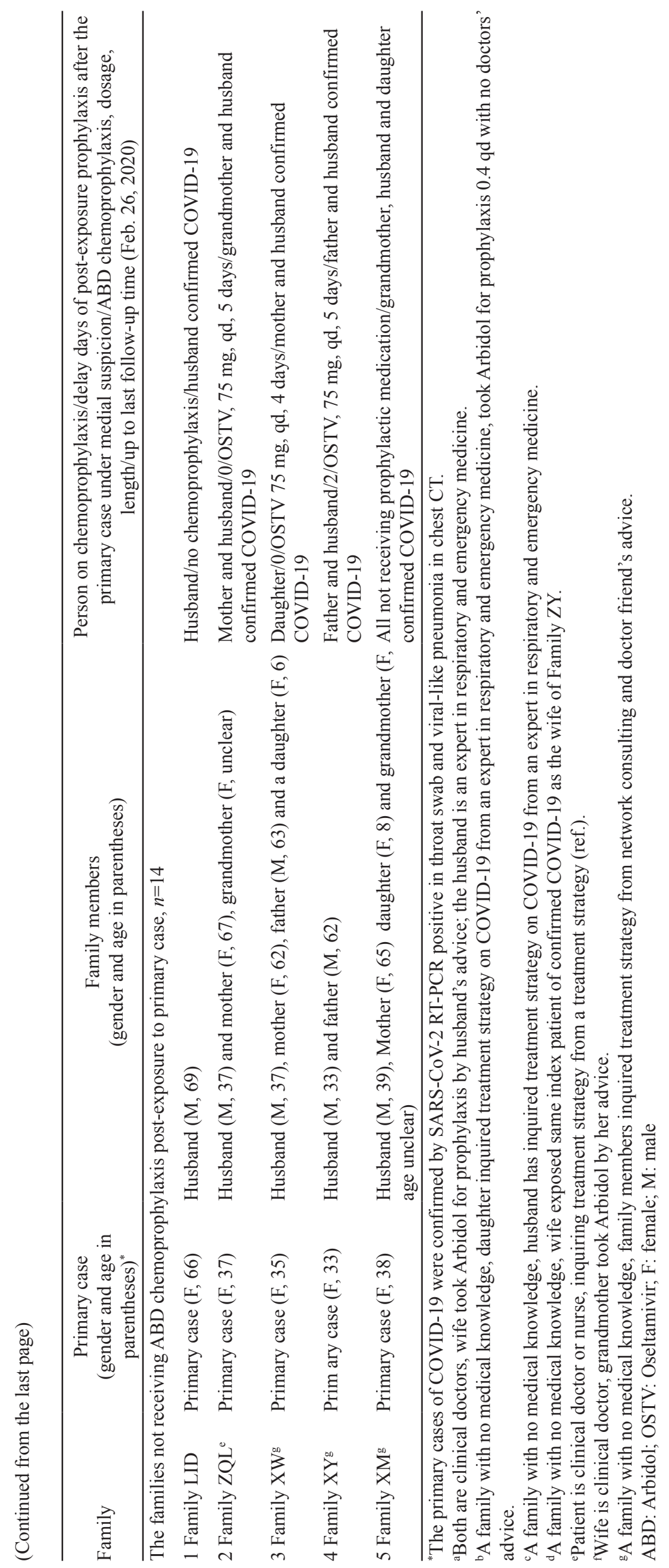


Table 2 Demographic data and characteristics of the subjects

\begin{tabular}{|c|c|c|c|c|c|c|}
\hline \multirow{2}{*}{ Parameters } & \multicolumn{3}{|c|}{ Health care workers $(n=124)$} & \multicolumn{3}{|c|}{ Family members $(n=66)$} \\
\hline & Arbidol $(n=55)$ & No Arbidol $(n=69)$ & $P^{*}$ & Arbidol $(n=45)$ & No Arbidol $(n=21)$ & $P^{*}$ \\
\hline$\overline{\text { Age (years), mean } \pm \mathrm{SD}}$ & $35.5 \pm 7.9$ & $33.4 \pm 7.1$ & & $40.7 \pm 18.6$ & $40.1 \pm 23.8$ & \\
\hline Age stratification & & & 0.215 & & & 0.628 \\
\hline$<34$ & $29(52.7)$ & $44(63.8)$ & & $13(28.9)$ & $6(28.6)$ & \\
\hline$\geq 34$ & $26(47.3)$ & $25(36.2)$ & & $28(62.2)$ & $11(52.4)$ & \\
\hline Unknown & $0(0.0)$ & $0(0.0)$ & & $4(8.9)$ & $4(19.0)$ & \\
\hline Adulthood & & & & & & 0.057 \\
\hline Yes & $55(100.0)$ & $69(100.0)$ & & $39(86.7)$ & $14(66.7)$ & \\
\hline No & $0(0.0)$ & $0(0.0)$ & & $6(13.3)$ & $7(33.3)$ & \\
\hline Gender, $n(\%)$ & & & $0.002^{\#}$ & & & 0.252 \\
\hline Male & $17(30.9)$ & $6(8.7)$ & & $19(42.2)$ & $10(47.6)$ & \\
\hline Female & $38(69.1)$ & $63(91.3)$ & & $24(53.3)$ & $7(33.3)$ & \\
\hline Unknown & $0(0.0)$ & $0(0.0)$ & & $2(4.4)$ & $4(19.0)$ & \\
\hline Occupation & & & $<0.001^{\#}$ & & & \\
\hline Doctor & $29(52.7)$ & $11(15.9)$ & & $1(2.2)$ & $1(4.8)$ & \\
\hline Nurse & $26(47.3)$ & $58(84.1)$ & & $0(0.0)$ & $0(0.0)$ & \\
\hline Others & $0(0.0)$ & $0(0.0)$ & & $44(97.8)$ & $20(95.2)$ & \\
\hline Working place & & & $<0.001^{\#}$ & NA & NA & NA \\
\hline Fever clinic & $19(34.5)$ & $3(4.3)$ & & NA & NA & NA \\
\hline IPD" & $21(38.2)$ & $55(79.7)$ & & NA & NA & NA \\
\hline Quarantine ward & $15(27.3)$ & $11(15.9)$ & & NA & NA & NA \\
\hline Chronic comorbidities ${ }^{\S}$ & & & 0.431 & & & 0.659 \\
\hline Yes & $2(3.6)$ & $1(1.4)$ & & $6(13.3)$ & $2(9.5)$ & \\
\hline No & $53(96.4)$ & $68(98.6)$ & & $39(86.7)$ & $19(90.5)$ & \\
\hline $\begin{array}{l}\text { Incubation time (days) } \\
\text { Median (range) }\end{array}$ & $4(4--4)$ & $13(4-24)$ & & $5(5-5)$ & $4(2-20)$ & \\
\hline $\begin{array}{l}\text { Observation time (days) } \\
\text { Median (range) }\end{array}$ & $43(19-59)$ & $50(22-59)$ & & $33(25-42)$ & $37(28-40)$ & \\
\hline
\end{tabular}

"chi square test; ${ }^{\#} P<0.05$

"IPD: inpatient department; NA: not applicable; incubation time: onset time-exposure time (only application to infected patients); observation time: last follow-up time-exposure time (only application to uninfected persons); \$ hypertension, diabetes, vascular disease, obesity (body mass index $>30$ )

Table 3 Correlation between Arbidol and no Arbidol for prevention of COVID-19 infection in family clusters

\begin{tabular}{lccccc}
\hline Treatment & & COVID-19 infection & No infection & HR $(95 \% \mathrm{CI})^{*}$ & $P^{*}$ \\
\hline Arbidol, $n(\%)$ & No & $12(92.3)$ & $9(17.4)$ & 1.00 (Reference) & \\
& Yes & $1(7.7)$ & $44(83.0)$ & $0.025(0.003-0.209)$ & $0.0006^{\#}$ \\
\hline
\end{tabular}

${ }^{*}$ Cox regression calculations, gender, age, occupation, basic physical condition and working place adjusted.

${ }^{*} P<0.05 . n$ : number; HR: Hazard ratio

Table 4 Correlation between Arbidol and no Arbidol for prevention of COVID-19 infection in health care workers

\begin{tabular}{lccccc}
\hline Treatment & & COVID-19 infection & No infection & HR $(95 \% \mathrm{CI})^{*}$ & $P^{*}$ \\
\hline Arbidol, $n(\%)$ & No & $7(87.5)$ & $62(53.4)$ & 1.00 (Reference) & \\
& Yes & $1(12.5)$ & $54(46.6)$ & $0.056(0.005-0.662)$ & $0.0221^{\#}$ \\
\hline
\end{tabular}

${ }^{*}$ Cox regression calculations, gender, age, occupation, basic physical condition and working place adjusted. ${ }^{\#} P<0.05$. $n$ : number. HR: Hazard ratio

\subsection{Cox Proportional Hazards Model}

The Cox proportional hazards model (Cox regression) demonstrated that Arbidol was a protective factor against COVID-19 infection [hazard ratio (HR) $0.025,95 \%$ CI $0.003-0.209, P=0.0006$ in family members and HR $0.056,95 \%$ CI $0.005-0.662, P=$ 0.0221 in health care workers] (table 3 and table 4 ) after adjusting the gender, age, workplace, occupation and basic physical condition.

\section{DISCUSSION}

Wuhan Union Hospital is the first hospital to recommend compassionate prescription of Arbidol for patients with confirmed or suspected COVID-19 in China. The early release of Therapeutic and Triage Strategy for 2019 Novel Coronavirus in Wuhan Union Hospital and the publication of it later on ${ }^{[10]}$ helped health care workers to figure out a possible way for 
PEP and this information was transmitted to some community families directly or indirectly through the health care workers in this hospital.

Arbidol showed activity to coronavirus in vitro ${ }^{[10]}$. Our study may provide clinical support for Abidol's potential for preventing transmission of SARS-Cov-2. The low HR for the family members and the health care workers on Arbidol PEP suggests a protective effect of Arbidol against COVID-19 transmission. There were no differences in dose, the length of days of the medication, delay time of PEP after the diagnosis of primary case in family and health care workers cohorts with or without Arbidol PEP (data were not shown, $P>0.05$ ), which may be related to the fact that $75 \%$ of Arbidol users took the prescribed dose according to instructions and the duration of administration for $91 \%$ of Arbidol users was at least the recommended time in the label instruction $(0.2 \mathrm{~g}$, tid for 5 days).

Work location played a significant role in disease transmission $(P<0.001)$. The neurology department where the cluster of health care worker infections began is located in the internal medicine building, which is home to 800 medical beds. Most of health care workers who did not take Arbidol worked in the inpatient department, where protection protocol was less strict than in the fever clinic and quarantine ward due to the shortage of epidemic prevention materials. The difference in gender and professional distribution of health care personnel may be due to the fact that our participants included more female nurses ( 80 female nurses of 101 female healthcare workers).

As a preliminary report, our study has limitations. Firstly this study is a retrospective study and includes potential selection bias. Secondly, we did not assess the awareness level of the family members towards Arbidol PEP. Thirdly, due to the medical demand and surge in the number of patients in the early stage of the outbreak, we did not have enough time for virus sequence detection.

Our results show the association between Arbidol PEP and prevention of COVID-19 transmission is significant. A well-designed, large scale prospective study and/or randomized controlled trial is necessary to further validate the use of Arbidol for post-exposure prophylaxis.

\section{Conflict of Interest Statement}

We declare no competing interests.

\section{REFERENCES}

1 Guan W, Ni Z, Hu Y, et al. Clinical characteristics of 2019 novel coronavirus infection in China. medRxiv preprint, 2020.

2 Chan JF, Yuan S, Kok KH, et al. A familial cluster of pneumonia associated with the 2019 novel coronavirus indicating person-to-person transmission: a study of a family cluster. Lancet, 2020, 395(10223):514-523

3 Uyeki TM, Bernstein HH, Bradley JS, et al. Clinical Practice Guidelines by the Infectious Diseases Society of America: 2018 Update on Diagnosis, Treatment, Chemoprophylaxis, and Institutional Outbreak Management of Seasonal Influenza. Clin Infectious Dis, 2019,68(6):E1-E47

4 Pecheur EI, Borisevich V, Halfmann P, et al. The Synthetic Antiviral Drug Arbidol Inhibits Globally Prevalent Pathogenic Viruses. J Virol, 2016,90(6):30863092

5 Brooks MJ, Burtseva EI, Ellery PJ, et al. Antiviral Activity of Arbidol, a Broad-Spectrum Drug for Use Against Respiratory Viruses, Varies According to Test Conditions. J Med Virol, 2012,84(1):170-181

6 Ji X, Zhao Y, Zhang M, et al. The experimetal study of the anti-SARS-Cov effect of arbidol. J Pharm PLA (Chinese), 2004,20(04):274-276

7 Khamitov RA, Loginova S, Shchukina VN, et al. Antiviral activity of arbidol and its derivatives against the pathogen of severe acute respiratory syndrome in the cell cultures. Vopr Virusol, 2008, 53(4):9-13

8 Chen N, Zhou M, Dong X, et al. Epidemiological and clinical characteristics of 99 cases of 2019 novel coronavirus pneumonia in Wuhan, China: a descriptive study. Lancet, 2020,395(10223): 507-513

9 Wang D, Hu B, Hu C, et al. Clinical Characteristics of 138 Hospitalized Patients with 2019 Novel CoronavirusInfected Pneumonia in Wuhan, China. JAMA, 2020

10 Zhang J, Zhou L, Yang Y, et al. Therapeutic and triage strategies for 2019 novel coronavirus disease in fever clinics. Lancet Respir Med, 2020,8(3):e11-e12

(Received Apr. 15, 2020; revised May 4, 2020) 